\title{
Individual Packet Deadline Constrained Opportunistic Scheduling For a Multiuser System
}

\author{
M. Majid Butt, Kimmo Kansanen, Ralf R. Müller \\ Institute of Electronics and Telecommunications \\ Norwegian University of Science and Technology, Trondheim, Norway \\ Email: majid.butt@iet.ntnu.no
}

\begin{abstract}
In this work an opportunistic scheduling scheme is presented and analyzed for a multiuser system. The objective of the proposed scheme is to minimize the system transmit energy in the presence of a hard deadline delay constraint for the individual packets. In the large system limit, the scheme is modeled and analyzed in the scenario when arriving packets have associated deadlines which vary from packet to packet. We introduce transmission thresholds that depend on channel quality and number of time slots left before a packet reaches its hard deadline. These thresholds are optimized such that they reflect the interaction of deadline delay and channel variation, and result in a minimum system energy. The results demonstrate the saving in energy for a system where the applications have individual packet deadline delay constraints.
\end{abstract}

\section{INTRODUCTION}

In modern wireless systems, delay guarantees for data communications are becoming more and more important. These guarantees take the form of either average delay or strict deadline for transmission. For example, multimedia applications require strict deadline guarantees as compared to some of the data transfer applications where soft average delay requirements are sufficient to be fulfilled. On the other hand, with the amount of multimedia traffic added in a modern communication network, provision of hard deadline is getting tougher. From a service provider's point of view, this task needs to be accomplished with minimal energy resources.

In [1], Knopp and Humblet proposed a scheme which maximizes the information capacity by scheduling the user with the highest channel quality. In [2], Proportional Fair Scheduling (PFS) was proposed to provide fairness guarantees to all the users. Neely discussed an optimal scheduler when inter-arrival and inter-transmission time have some asymmetry property in [3]. Reference [4], [5] discuss formulation of data transmission for deadline constrained systems and propose energy efficient schedulers.

In [6] Sequential Deadline Dependent Partial buffer Scheduling (SDDPS) scheme has been proposed for a deadline constrained multiuser system with a constant arrival process. SDDPS has been analyzed for random arrivals in [7] for the case when all the arriving packets have identical deadlines. This work generalizes the results in [6], [7] for the case when the arriving packets have individual, non-identical deadlines. The main contribution of this work is modeling and analysis of opportunistic scheduler for the non-identical deadline case. We prove that to each system with non-identical deadlines, there exists an equivalent system with identical deadlines in the large system limit.

The rest of the paper is organized as follows. In Section II, system model for this work is described. Section III introduces a state space description for the proposed scheduling scheme. In Section IV, the large system analysis of the scheme is presented. Section V shows numerical results for the proposed scheme and Section VI concludes with the summary of the main contributions of this paper.

\section{SySTEM MODEL}

We consider a multiple-access system with $K$ users randomly placed within a certain geographical area. Each user requires an average rate $R$ equals to $\frac{\Gamma}{K}$ where $\Gamma$ denotes the spectral efficiency of the system measured in bits/s/Hz. We assume a time-slotted system. In each time slot, arrivals are random and we model them as constant arrivals with variable size [7]. We consider an uplink scenario but the results can be generalized to the downlink as well.

Each user experiences a distance dependent path loss and an environment-dependent fading. The channel gain $g_{k}(t)$ is the product of path loss $s_{k}$ and short-term fading $f_{k}(t)$ i.e. $g_{k}(t)=s_{k} f_{k}(t)$. Path loss and short-term fading are assumed to be independent. The path loss is a function of the distance between the transmitter and the receiver and remains constant within the time-scales considered in this work. For a multiband system of $M$ channels, each user decides to transmit on its best channel. Short-term fading over the best channel is represented by $f_{k}(t)=\max \left(f_{k}^{(1)}(t), f_{k}^{(2)}(t), \ldots, f_{k}^{(M)}(t)\right)$. Short-term fading depends on the scattering environment and varies from slot to slot for every user. It is independent and identically distributed across both users and slots, but remains constant within each time slot.

$E_{k}^{R}(t)$ and $E_{k}(t)$ represent the received and the transmitted energy for each user $k$ such that

$$
E_{k}^{R}(t)=g_{k}(t) E_{k}(t) .
$$

Note that the distribution of $g_{k}(t)$ differs from user to user.

Let $N_{0}$ denote the noise power spectral density. We allow scheduling of multiple users in the same frequency band. 
Simultaneously scheduled users are separated by superposition coding. Channel state information is assumed to be known at both the transmitter and the receiver side.

Let $\Delta_{m}$ be the set of users to be scheduled in frequency band $m . \pi_{k}^{(m)}$ denotes the permutation of the scheduled user indices for frequency band $m$ that sorts the channel gains in increasing order, i.e. $g_{\pi_{1}}^{(m)} \leq \cdots \leq g_{\pi_{k}}^{(m)} \leq \cdots \leq d_{\pi_{\left|\Delta_{m}\right|}}^{(m)}$. Then, the energy of the user $\pi_{k}^{(m)}$ with rate $R_{\pi_{k}}^{(m)}$, as scheduled by the scheduler to guarantee an error free communication, is given by [8], [9]

$$
E_{\pi_{k}}^{(m)}=\frac{N_{0}}{g_{\pi_{k}}^{(m)}}\left[2^{\sum_{i \leq k} R_{\pi_{i}}^{(m)}}-2^{\sum_{i<k} R_{\pi_{i}}^{(m)}}\right] .
$$

Eq. (2) represents the minimum total transmit energy assignment for the scheduled users.

\section{SDDPS With PaCKets DePendent Deadlines}

We model the SDDPS scheduler by a Markov process. The maximum possible deadline of an arriving packet in the buffer is denoted by $n$. Then, we define the deadline of an arriving packet $q<n$ with respect to $n$. i.e. the deadline of a packet with deadline of $0 \leq a<n$ time slots less than $n$ is represented by $q=n-a$ where $a$ is termed as deadline offset. We denote probability of arrival of a packet with deadline $q$ by $p_{q}$. We assume infinite size buffer for simplicity of analysis.

We model the arrival of a packet with deadline $q=n-a$ by a packet that already has spent $a$ time slots in the buffer. By this initial offsetting, we model a system with non-identical packet deadlines equivalent to a system with identical packet deadlines. We define some terms used in the work.

Definition 1 (Deadline Distance): The deadline distance $\nu \in\{1 \ldots n\}$ for a packet is defined as the number of time slots remaining before it reaches its hard deadline.

Note that deadline distance for an arriving packet equals $q$.

Definition 2 (Backlog State): Backlog state $i$ in a Markov chain is defined as the minimum of the deadline distance for the packets waiting to be scheduled in the buffer.

$$
i=\min \left(\nu_{1}, \nu_{2} \ldots \nu_{j}\right)
$$

where $\nu_{j}$ represents the deadline distance of the $j$ th packet in the buffer. For simplicity, we denote backlog state by a state.

Definition 3 (Transmission threshold:): A transmission threshold $\kappa_{i \rightarrow j}$ is defined as the minimum short-term fading value allowing for the transition $T_{i \rightarrow j}$ from state $i$ to state $j$.

We consider the case when arriving packets have nonidentical individual hard deadlines. In SDDPS, a group of users having better fading than the transmission threshold are scheduled for transmission simultaneously. All the state transitions have associated thresholds. Each user compares the current short-term fading $f_{k}$ with the threshold $\kappa_{i \rightarrow j}$ for every state $j \geq i$ sequentially. The thresholds are computed such that the transmission threshold is minimum for the state closest to the deadline. If $f_{k}>\kappa_{i \rightarrow i}$, all the packets with deadline distance $\nu_{i}$ are scheduled for transmission and the threshold of the next higher state is compared with $f_{k}$. The transmission threshold for the state $i-1$ will be greater than the transmission threshold of the state $i$ as the packet in this state has deadline distance $\nu$ one more than the deadline distance of the scheduled packet. Similarly, the thresholds of all the states $j \geq i$ are compared sequentially with $f_{k}$ until $f_{k}$ is less than the threshold $\kappa_{i \rightarrow j+1}$ of a state. The last state in which a packet is scheduled is termed as ending state $j(t)$. The scheduler moves to state $j(t)$ from a state $i(t)$ by scheduling packets in the $L$ intermediate states and therefore, ending state $j(t)$ is given by

$$
j(t)=i(t)+L(y, i)-1
$$

The state $i(t+1)$ is determined after the arrival of a new packet at time $t+1$. Depending on the deadline of the next arriving packet, the scheduler stays in state $j(t)$ or moves into state $q(t+1)$ such that

$$
i(t+1)=\left\{\begin{array}{lll}
j(t) & \text { if } & q(t+1) \geq j(t) \\
q(t+1) & \text { if } & q(t+1)<j(t)
\end{array}\right.
$$

where $q(t+1)$ denotes the deadline offset of the arriving packet at time $t+1$. If $f_{k}$ is less than the thresholds of all the states, no packet is scheduled. Then, the scheduler moves into state $i(t+1)$ according to the Eq. (5) where $j(t)=i(t)-1$. The deadline distance $\nu$ of all the remaining un-scheduled packets in the buffer is decremented by one in each time slot.

We model the special case when no packet arrives in a time slot by considering arrival of a packet with zero size and deadline $n$. This assumption keeps our state space description consistent for no arrival case as well. When a packet with zero size is scheduled for transmission, no rate is allocated.

State transition is a two step process. The scheduler schedules packets in $L(y, i)$ states, allocates rate for the actual data and then moves into state $i(t+1)$ according to Eq. (5). Note that the scheduler schedules packets in $L(y, i)$ states without the knowledge that arriving packets have identical or nonidentical deadlines. It is a packet based scheduling algorithm and the transmission thresholds depend only on the fading distribution and number of time slots left before the deadline.

State transition diagram of the SDDPS scheduler with packets having non-identical deadlines is shown in Fig. 1.

For identical deadline case, State Transition Matrix (STM) is given by [6]

$$
\mathbf{P}_{\text {SDDPS }}^{\mathrm{id}}=\left(\begin{array}{cccc}
\alpha_{11} & \alpha_{12} & \cdots & \alpha_{1 n} \\
\ddots & \ddots & \ddots & \ddots \\
0 & \cdots & \alpha_{(n-1)(n-1)} & \alpha_{(n-1) n} \\
0 & \cdots & \alpha_{n(n-1)} & \alpha_{n n}
\end{array}\right) .
$$

where $\alpha_{i j}$ is defined as

$$
\alpha_{i j}=\operatorname{Pr}\left(\kappa_{j}<f \leq \kappa_{j+1}\right) .
$$

The termination condition $\kappa_{i \rightarrow n+1}$ is defined as $\infty$. We set $\kappa_{1 \rightarrow 1}$ to zero to ensure the transmission of the packet reaching its deadline.

To compute STM for the non-identical deadline case, we evaluate the effect of non-identical deadlines on state space 


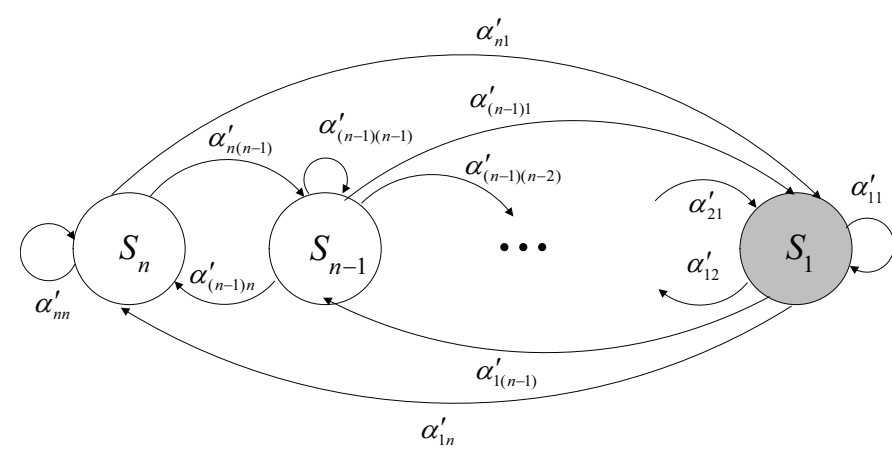

Fig. 1. State diagram for the transition states for the SDDPS scheduler.

representation. In the first step, we compute the value of $j$ as described in Eq. (7) for the identical deadline case and remains the same for non-identical deadline case. However, STM for non-identical case is modified by the deadline distribution as well. In the second step, for a given pair of $i, j$, we compute the offset produced by this distribution by evaluating the offset matrix $\mathbf{S}^{\text {nid }}$ given by

$$
\mathbf{S}^{\mathrm{nid}}=\left(\begin{array}{cccc}
\sum_{\mu=1}^{n} p_{\mu} & 0 & \ldots & 0 \\
p_{1} & \sum_{\mu=2}^{n} p_{\mu} & \cdots & 0 \\
\ddots & \ddots & \ddots & \ddots \\
p_{1} & \cdots & p_{n-1} & \sum_{\mu=n}^{n} p_{\mu}
\end{array}\right) .
$$

where probability $S^{\text {nid }}(q, j)$ is defined as

$$
S^{\text {nid }}(q, j)=\left\{\begin{array}{lll}
0 & \text { if } & q>j \\
\sum_{\mu=j}^{n} p_{\mu} & \text { if } & q=j \\
p_{q} & \text { if } & q<j
\end{array}\right.
$$

Diagonal elements in Eq. (8) represent the sum of probabilities of deadline distribution which keeps $i(t+1)=j(t)$ while non zero non diagonal elements represent the probability when $i(t+1)=q(t+1)$ as explained in Eq. (5). We can represent STM for non identical case as a product of Eq. (6) and Eq. (8) such that

$$
\mathbf{P}_{\mathrm{SDDPS}}^{\mathrm{nid}}=\mathbf{P}_{\mathrm{SDDPS}}^{\mathrm{id}} \mathbf{S}^{\text {nid }}
$$

One obvious difference between the STMs for the systems having arrived packets with identical deadlines and the systems having arrived packets with non-identical deadlines is the transition probabilities when no packet is scheduled. If all the arriving packets have identical deadline of $n$ time slots, the only possible state is $i-1$. However, if the deadlines are non identical, depending on the deadline of the arriving packet, the scheduler moves into any of the states $j<i$.

Example: We explain it with the help of an example when $n=2$. If all the packets have identical deadline, $\mathbf{P}_{\text {SDDPS }}^{\text {id }}$ is given by

$$
\mathbf{P}_{\text {SDDPS }}^{\mathrm{id}}=\left(\begin{array}{ll}
\alpha_{11} & \alpha_{12} \\
\alpha_{21} & \alpha_{22}
\end{array}\right) .
$$

If the packets have non-identical deadlines of $n_{1}=1$ and $n_{2}=2, n$ equals 2 and resulting $\mathbf{P}_{\mathrm{SDDPS}}^{\mathrm{nid}}$ is given by

$$
\mathbf{P}_{\mathrm{SDDPS}}^{\mathrm{nid}}=\left(\begin{array}{ll}
\alpha_{11}+p_{1} \alpha_{12} & p_{2} \alpha_{12} \\
\alpha_{21}+p_{1} \alpha_{22} & p_{2} \alpha_{22}
\end{array}\right) .
$$

Note that Eq. (12) is reduced to Eq. (11) if all the arriving packets have identical deadline of 2 .

\section{ASYMPTOTIC ANALYSIS OF SDDPS For Non-Identical DeAdline CASE}

In this section, we analyze SDDPS in the large system limit. In Section V, numerical results show that as number of users increases, the results follow the asymptotic results closely. We use the large system results from [9]. We model a user that sends $L$ packets at a time as $L$ users with identical fading that transmit a single packet and call them virtual users. For analysis purpose, we use the term scheduled virtual users (SVU) for the scheduled packets in this section. The average energy consumption of the system per transmitted information bit at the large system limit $K \rightarrow \infty$ is then given by

$$
\left(\frac{E_{\mathrm{b}}}{N_{0}}\right)_{\mathrm{sys}}=\log (2) \int_{0}^{\infty} \frac{2^{R \mathrm{P}_{d, \mathrm{SVU}}(x)}}{x} \mathrm{dP}_{d, \mathrm{SVU}}(x)
$$

where $\mathrm{P}_{d, \mathrm{SVU}}(\cdot)$ is the the cumulative distribution function (cdf) of the fading of the SVUs.

$\mathrm{P}_{d, \mathrm{SVU}}(x)$ is composed of the short-term fading and the long-term fading of the SVUs. In the large system limit, the long-term fading of the SVUs follows the same distribution as the long-term fading of all users because long-term and short-term fading are mutually statistically independent and state transitions depend only on the short-term fading. The probability density function (pdf) of the short-term fading of the scheduled virtual users is given by

$$
\mathrm{p}_{f, \mathrm{SVU}}(y)=\sum_{i=1}^{n} \pi_{i} \mathrm{p}_{f, \mathrm{SVU}}(y \mid i)
$$

where $\pi_{i}$ denotes the limiting probability of state $i$ and the channel distribution of the users in state $i$ is given by

$$
\mathrm{p}_{f, \mathrm{SVU}}(y \mid i)=c_{i}^{n i d} L(y, i) \mathrm{p}_{\max \{f\}}(y)
$$

with $\mathrm{p}_{\max \{f\}}(y)$ and $c_{i}^{\text {nid }}$ denote the short-term fading of the best of the channels for a multi-channel system and a constant to normalize the conditional pdf.

We represent the channel distribution of SVUs in terms of weighted sum of rate allocation for probabilistic state transitions. Note that scheduled rate is only a function of fading and state as shown in Eq. (7), but deadline distribution of the arriving packets randomizes the state transition and corresponding rate allocation function. For a given fading $f$, rate is probabilistically allocated to the states $i \leq r \leq j$ and weighted by the probability of ending up in state $r$. Note that probability of ending in state $r$ can directly be computed from Eq. (9) for given $i, j$. We can write $\mathrm{p}_{f, \mathrm{SVU}}(y \mid i)$ as

$$
\begin{aligned}
\operatorname{p}_{f, \mathrm{SVU}}(y \mid i) & =c_{i}^{n i d} \mathrm{E}\left(\mu_{t}\right)\left((j-i+1) \sum_{l=j}^{n} p_{l}\right. \\
& \left.+\sum_{r=i}^{j-1}(r-i+1) p_{r}\right) \operatorname{p}_{\max \{f\}}(y)
\end{aligned}
$$


where $j$ is uniquely defined by Eq. (7) and rate of arrival in each scheduled state is $\mathrm{E}\left(\mu_{t}\right)$.

We give a more intuitive description of the effect of nonidentical deadlines on system behaviour. Note that arrival of a new packet always takes place in state $n$ for the case when all the packets have identical deadlines. For a two state system with $n=2$, if a packet is not scheduled at time $t$, it moves into state 1 with probability one. In non-identical deadline case, the probability of arrival in state two is $p_{2}$. If a packet is not scheduled in state 2 , it moves into state 1 with probability $p_{2}$, making rate of arrival $p_{2}$ for state 1 . However, there is an additional direct source of arrival in state 1 with probability $p_{1}$ at time $t+1$ (due to deadline offset). Therefore, cumulative buffer content of state 1 is summation of $p_{1}$ and $p_{2}$ while buffer content of state 2 is $p_{2}$. Recall that arrival with nonidentical deadlines results in insertion of the arriving packet with deadline less than $n$ in state $q$. The resulting content size for the states with small deadline distances is greater as compared to the states with large deadline distances. For a state $r$, this effect is modeled in Eq. (17) by considering an equivalent arrival process whose arrival rate is a summation of the arrival probability $p_{l}$ over $r \leq l \leq n$. For a given $i, j$ pair, due to non-identical deadline of the arriving packets, the random content size in $L(y, i)$ scheduled states is given by

$$
L(y, i)=\sum_{r=i}^{j} \sum_{l=r}^{n} p_{l}
$$

Consequently, using Eq. (17) in Eq. (15), the channel distribution of the scheduled users for the non-identical deadline case can be written in terms of rate allocation function for a fixed state transition and random content, and given by

$$
\mathrm{p}_{f, \mathrm{SVU}}(y \mid i)=c_{i}^{n i d} \mathrm{E}\left(\mu_{t}\right) \sum_{r=i}^{j} \sum_{l=r}^{n} p_{l} \operatorname{p}_{\max \{f\}}(y)
$$

where $c_{i}^{n i d}$ is a normalization constant.

Lemma 1: The representations of distribution of scheduled virtual users in Eq. (16) and Eq. (18) are equivalent.

Proof of equivalence is omitted due to space limitations.

Note that there is an important difference between modeling of random arrival as constant arrival with random size in [7] and modeling packet arriving with non identical deadlines. In the limiting case, the content of every scheduled state converges to the rate of arrival process $E\left(\mu_{t}\right)$ for the modeling of random arrivals. However, contents of states are non uniform for non-identical packet deadline case and increase for the states closer to deadline. Random content size description helps in understanding the energy behaviour of the system when deadline constraints are non-identical for individual packets.

Corresponding channel distribution of the SVUs for the identical deadline case is given by

$$
\mathrm{p}_{f, \mathrm{SVU}}(y \mid i)=c_{i}^{i d} \mathrm{E}\left(\mu_{t}\right)(j-i+1) \mathrm{p}_{\max \{f\}}(y)
$$

where $c_{i}^{i d}$ is a normalization constant.
Although, non-identical deadline case has been modeled as an extension of identical deadline case, state space description and channel distributions are not equivalent due to different STMs. However, as SDDPS scheduler treats every packet individually based on its deadline distance, transmission thresholds optimized for the systems with identical deadlines remain optimal for the systems with non-identical deadlines as well.

\section{A. Optimization of Transmission Thresholds}

The optimization technique for the thresholds has been presented in [6] when all the arriving packets have identical deadlines. From analysis in Section IV, we conclude that the transmission thresholds depend only on the fading and current state (Eq. (7)), and remain the same as for the identical deadline case. In this section, we present the optimization procedure briefly for the completeness of discussion.

In SDDPS, for a single packet, a decision to wait in the buffer and wait for the next time slot has two effects. It gives the user a chance to transmit on the best channel out of $n$ opportunities available before the deadline. On the other hand, a forced transmission is costly. Transmission thresholds are designed to reflect these two effects.

As SDDPS treats each packet individually, regardless of state $i$, a packet with deadline distance $\nu_{j}$ requires the same transmission threshold for its transmission. For a packet with deadline distance $\nu_{j}$, we have

$$
\kappa_{j}=\kappa_{i \rightarrow j} \quad \forall i, j
$$

Eq. (20) helps us to reduce number of transmission thresholds from $\mathcal{O}\left(n^{2}\right)$ to $\mathcal{O}(n)$. For a deadline of $n$ time slots, we require $n-1$ optimized transmission threshold. We denote $\kappa_{i \rightarrow j}$ by $\kappa_{j}$ in rest of this work.

The optimized threshold vector is found using a recursive procedure explained in the following:

1) Start the optimization procedure for $n=2$ such that the optimization is a scalar problem and we only need to find the threshold $\kappa_{n}$ since $\kappa_{1}=0$.

2) Given the optimized threshold vector for $n$, i.e. $\vec{\kappa}^{\mathrm{opt}}(n)=\left[\kappa_{n}^{\mathrm{opt}}(n), \kappa_{n-1}^{\mathrm{opt}}(n), \ldots, \kappa_{2}^{\mathrm{opt}}(n), 0\right]$, we find the threshold vector for buffer length $n+1$ by the heuristic postulate $\vec{\kappa}(n+1)=\left[\kappa_{n}(n+1), \vec{\kappa}^{\mathrm{opt}}(n)\right]$ and optimize over $\kappa_{n}(n+1)$. Again, this is a scalar optimization problem.

Numerical results in [6], [7] provide evidence that transmission thresholds optimized using constraint in Eq. (20) and recursive procedure give comparable performance to the performance obtained by optimizing $\mathcal{O}\left(n^{2}\right)$ thresholds using more complex algorithms like simulated annealing.

\section{NumericAl Results}

We consider a multi-access channel with $M$ bands and it is assumed that fading on these channels is statistically independent. Every user senses $M$ channels and selects its best channel as a candidate for the transmission. We consider $M=10$ in our numerical results. We consider a system where users are placed uniformly at random in a cell except for a 
TABLE I

RECURSIVE THRESHOLD COMPUTATION AT $C=0.5 \mathrm{bits} / \mathrm{s} / \mathrm{Hz}$

\begin{tabular}{|c||c||c||c||c|}
\hline$n$ & $\kappa_{n}$ & $\kappa_{n-1}$ & $\kappa_{n-2}$ & $\kappa_{n-3}$ \\
\hline 2 & $\mathbf{2 . 5}$ & 0 & NA & NA \\
3 & $\mathbf{3 . 0}$ & 2.5 & 0 & NA \\
4 & $\mathbf{3 . 3}$ & 3.0 & 2.5 & 0 \\
\hline
\end{tabular}

forbidden region around the access point of radius $\delta=0.01$. The path loss exponent $\alpha$ equals 2 and path loss distribution follows the model described in [9]. All the users experience fast fading with exponential distribution with mean one on each of the $M$ channels.

The table I summarizes the threshold value vector $\vec{\kappa}$ for different values of $n$. The results are identical to the results in [6] where all the arriving packets have identical deadlines.

We show the convergence result of non-identical arrival case for the finite user population in Fig. 2. The figure shows the convergence of variance of the system energy for identical and non-identical deadline cases. We use Eq. (2) to evaluate system energy for finite number of users $K$. To compute the system energy for a specific number of users, 250 fading values have been used for a single path loss. The arrival process is Bernoulli with $p_{\text {arr }}=0.7$. The curves with $p_{1}=0$ and $p_{1}=1$ represent the identical deadline case when all the arriving packets have deadline 2 and 1 , respectively. $p_{1}=0.2$ represents the case when $20 \%$ of the packets arrive with $n=1$ and $80 \%$ with $n=2$. For all the cases, variance of the computed average system energy decreases as the number of users increases. The system energy for the system with a smaller deadline delay constraint converges faster as compared the distribution with a larger deadline delay. Hence, as ratio of $p_{1}$ decreases, it requires more number of users to converge.

Fig. 3 demonstrates the delay-energy trade off for a single channel system when the arriving packets have non-identical deadlines. We evaluate system performance at different spectral efficiencies. As the proportion of the packets with tight deadline constraint increases, average system energy increases correspondingly. However, this effect is more pronounced at small spectral efficiencies.

\section{CONCLUSions}

In this paper, we propose and analyze an opportunistic multiuser scheduling scheme for hard deadline delay constrained systems. The scheme is discussed in the realistic scenario when each arriving packet has individual deadline constraint. In the large system limit, the scheme is modeled and analyzed. We model the system having packets with nonidentical deadlines by offsetting their position in the buffer and show the difference in channel distributions of identical and non identical cases. The energy-delay tradeoff demonstrates the saving in energy for the delay tolerant applications. As the proportion of the packets with tight deadline constraint increases, the system transmit energy increases proportionally to reflect the loss due to forced deadline.

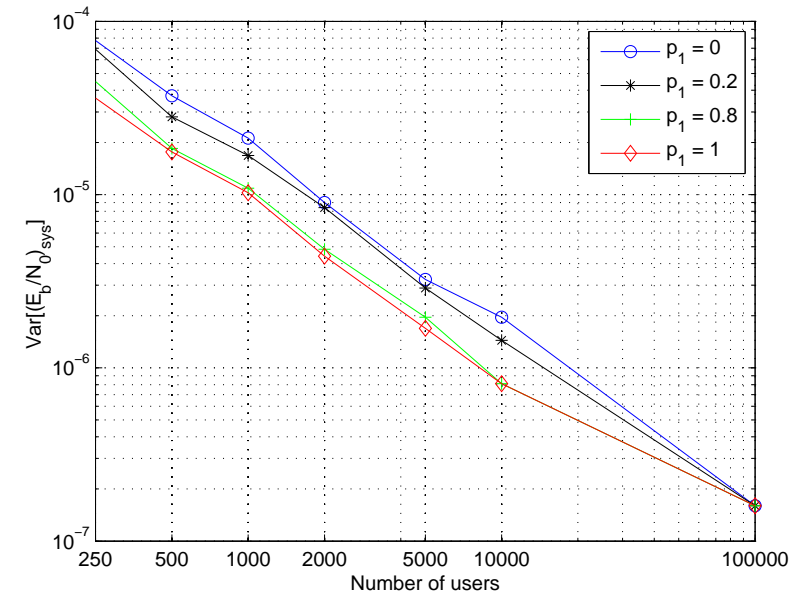

Fig. 2. Convergence of variance of System energy when arriving packets have deadline of 1 and 2 time slots.

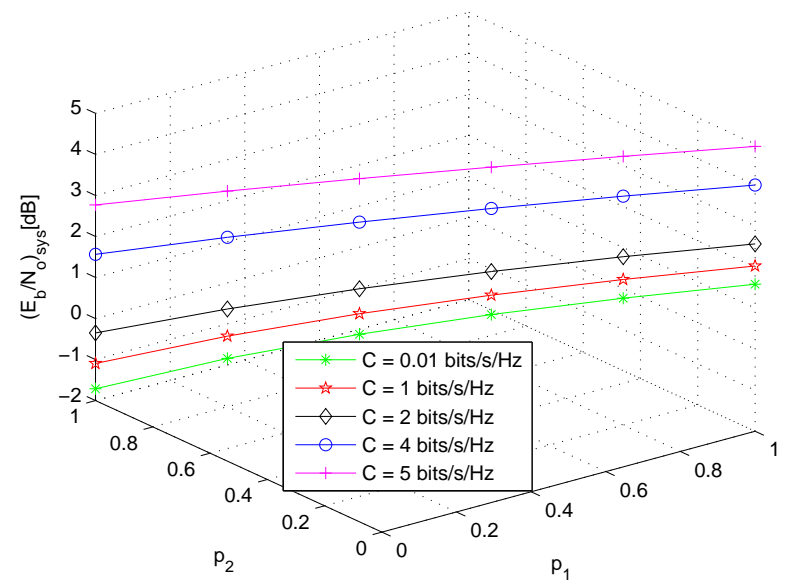

Fig. 3. The arriving packets have deadline of 1 and 2. The results demonstrate the effect when probability of packets with deadline one increases.

\section{REFERENCES}

[1] R. Knopp and P. Humblet, "Information capacity and power control in single cell multiuser communications," in IEEE, Int'l Conf. on Communications, June 1995.

[2] P. Viswanath, D. N. Tse, and R. Laroia, "Opportunistic beamforming using dumb antennas," IEEE Trans. Inform. Theory, vol. 46, no. 6, pp. 1277-1294, June 2002.

[3] W. Chan, M. J. Neely, and U. Mitra, "Energy-efficient transmissions with individual packet delay constraints," IEEE Trans. Inform. Theory, vol. 54, no. 5, pp. 2090 - 2109, May 2008.

[4] A. Tarello, J. Sun, M. Zafar, and E. Modiano, "Minimum energy transmission scheduling subject to deadline constraints," Wireless Networks, vol. 14 , no. 5, pp. 633-645, October 2008.

[5] J. Lee and N. Jindal, "Energy-efficient scheduling of delay constrained traffic over fading channels," IEEE Trans. Wireless Communications, vol. 8, no. 4, pp. 1866-1875, April 2009.

[6] M. M. Butt, K. Kansanen, and R. R. Müller, "Multiuser opportunistic scheduling for hard delay constrained systems," in IEEE Sarnoff Symposium 2008, Princeton, NJ, USA, April 2008.

[7] — "Hard deadline constrained multiuser scheduling for random arrivals," in WCNC, Cancun, Mexico, March 2011.

[8] S. Hanly and D. Tse, "Multi-access fading channels-part II: Delay limited capacities," IEEE Trans. Inform. Theory, vol. 44, no. 7, pp. 2816-2831, November 1998.

[9] G. Caire, R. Müller, and R. Knopp, "Hard fairness versus proportional fairness in wireless communications: The single-cell case," IEEE Trans. Inform. Theory, vol. 53, no. 4, pp. 1366-1385, April 2007. 